\title{
Formal synthesis of piperazinomycin, a novel antifungal antibiotic
}

\author{
Samir Ghosh, ${ }^{\text {a }}$ A. Sanjeev Kumar, ${ }^{\text {a }}$ G. N. Mehta, ${ }^{\text {b }}$ R. Soundararajan* a and Subhabrata \\ Sen $^{\mathbf{a}}$ \\ ${ }^{a}$ Chemical Research and Development Department, Pfizer Ltd, Mumbai-400705, India \\ ${ }^{b}$ Chemistry Section, Applied Sciences and Humanities Department, SVNIT, Surat-395 007, India \\ E-mail: soundara1959@gmail.com
}

\begin{abstract}
An alternative synthesis of piperazinomycin is disclosed. The approach is based on an intramolecular $\mathrm{O}$-arylation of arylboronic acid with phenol for formation of the macrocyclic biaryl ether.
\end{abstract}

Keywords: Piperazinomycin, antifungal, arylboronic acid, $O$-arylation

\section{Introduction}

Piperazinomycin 1, constitutes the simplest naturally occurring agent possessing the parent 14membered para- and metacyclophane diary1 ether structural subunit found in bouvardin, ${ }^{1}$ deoxybouvardin, ${ }^{1}$ RA-I-X, ${ }^{2}$ OF4949-I-OF494-IV, ${ }^{3}$ and K-13. ${ }^{4}$ This has renewed the interest in the synthesis and evaluation of piperazinomycin and structurally related agents since $\mathbf{1}$ and notably 2. However, efforts to critically examine the importance of the cycloisodityrosine subunit have been hampered by the synthetic inaccessibility of such systems.
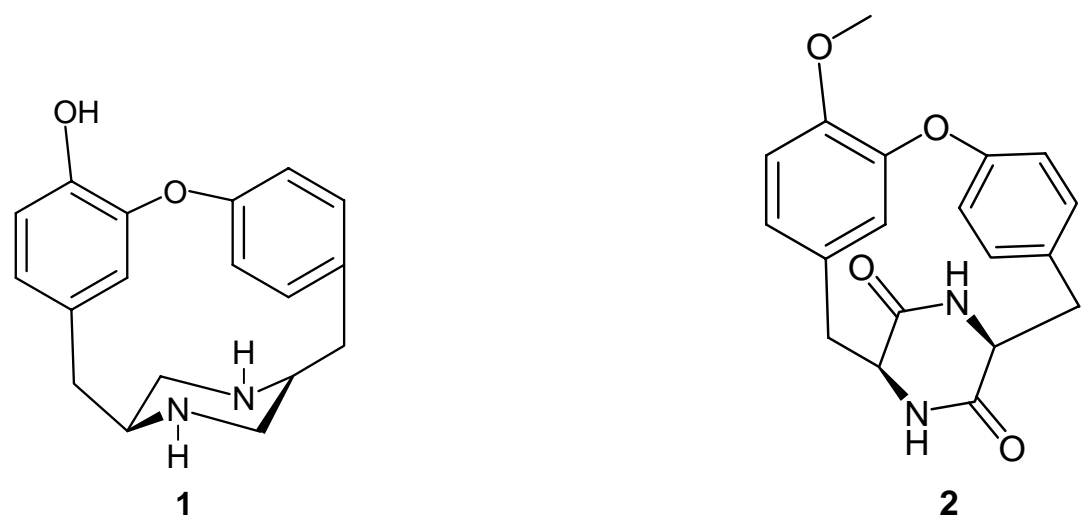


\section{Results and Discussion}

In the present paper, we describe a practical and short synthetic route to compound 2 from commercially available L-tyrosine by a coupling reaction of arylboronic acid with phenol originally developed by Chan, ${ }^{5}$ Evans, ${ }^{6}$ and Lam. ${ }^{7,8}$

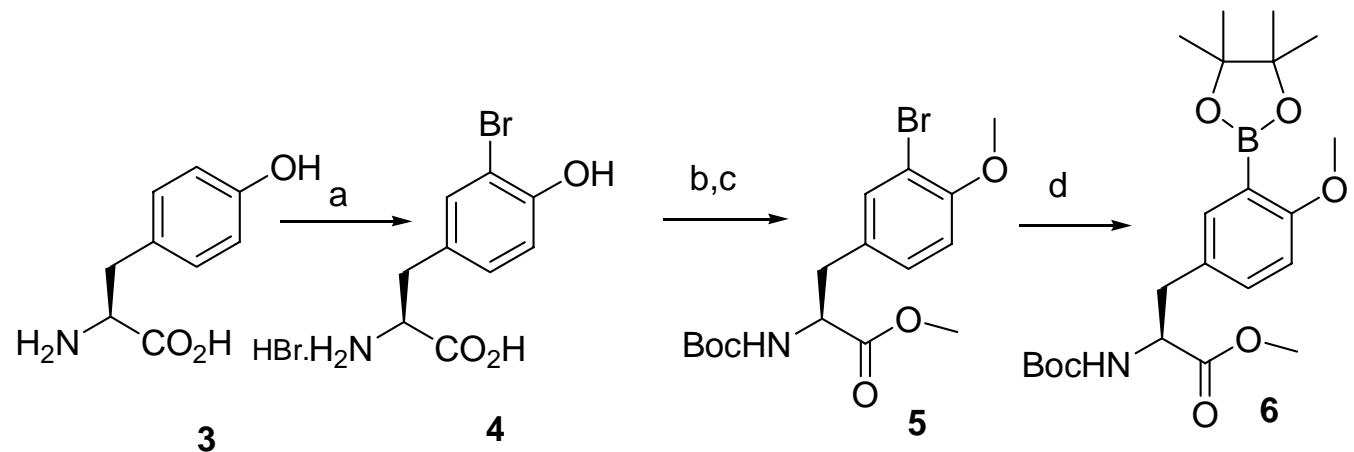

Scheme 1. (a) $\mathrm{Br}_{2} / \mathrm{AcOH}, \mathrm{HBr} / \mathrm{AcOH}, \mathrm{RT}, 6 \mathrm{~h}, 95 \%$; (b) (Boc) $)_{2} \mathrm{O}, \mathrm{NaHCO}_{3}, \mathrm{MeOH}, \mathrm{EtOAc}, \mathrm{RT}$, 4h (c) $\mathrm{Me}_{2} \mathrm{SO}_{4}, \mathrm{~K}_{2} \mathrm{CO}_{3}$, Acetone, RT, 12h, 96\% ( from 4 to 5) (d) Bis(pinacolato)diboron, KOAc, $\mathrm{PdCl}_{2}$ (dppf), DME, $110^{\circ} \mathrm{C}, 18 \mathrm{~h}, 90 \%$.

Commercially obtained L-tyrosine 3 was brominated at 3-position with bromine in acetic acid and hydrobromic acid in acetic acid at room temperature to afford 3-bromo- L-tyrosine hydrobromide $\mathbf{4}^{9}$ in $95 \%$ yield from 3 . Compound 4 was $N$-protected with a Boc group and methylated with dimethyl sulphate (2.5 eq.) in presence of potassium carbonate (3 eq.) in acetone to afford protected bromotyrosine 5 in $96 \%$ yield from 4 . Compound 5 was treated with bis (pinacolato) diboron (1.3 eq.), potassium acetate ( 3 eq.) and $\mathrm{PdCl}_{2}$ (dppf) (0.05 eq.), as a catalyst, in 1, 2-dimethoxy ethane (DME) at $110^{\circ} \mathrm{C}$ for $18 \mathrm{~h}$ to afford compound 6 in $90 \%$ yield.

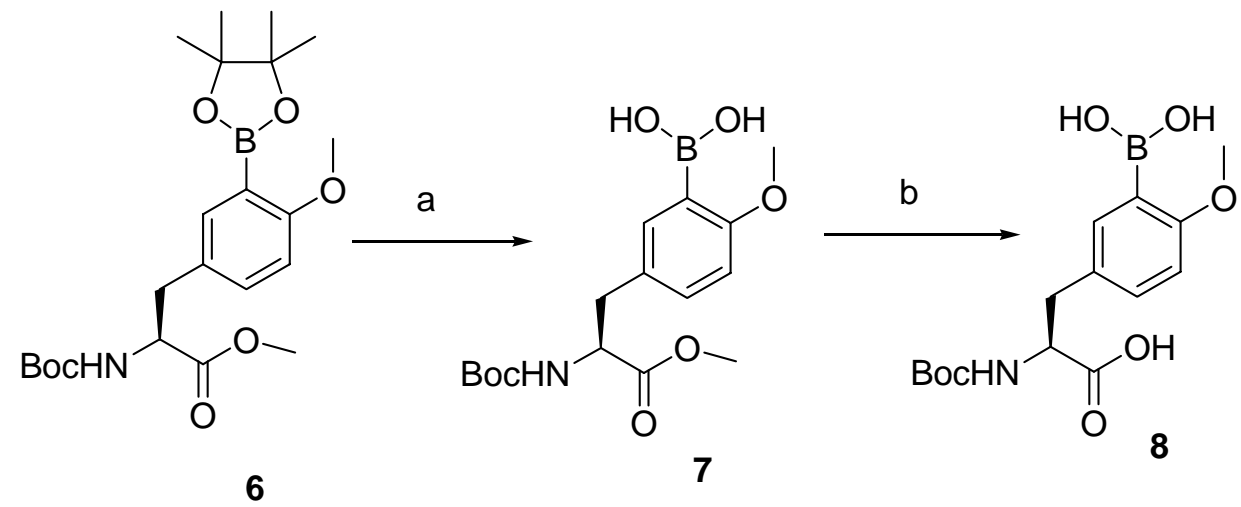

Scheme 2. (a) $\mathrm{NaIO}_{4}$, acetone, $0.1 \mathrm{~N}$ aq. $\mathrm{NH}_{4} \mathrm{OAc}$, RT, 16 h, 90 \%.(b) LiOH, $\mathrm{MeOH}, \mathrm{H}_{2} \mathrm{O}, \mathrm{RT}$, $30 \min , 96 \%$. 
The arylboronate 6 was easily converted into arylboronic acid $7^{10}$ using sodium metaperiodate (3 eq.) in acetone $(20 \mathrm{Vol})$ in presence of $0.1 \mathrm{M} \mathrm{NH} \mathrm{NH}_{4} \mathrm{OAc}$ aq. solution at room temperature for $16 \mathrm{~h}$. in $90 \%$ yield. Compound 7 was hydrolyzed with lithium hydroxide in methanol and water at room temperature for 30 min to afford compound 8 in $96 \%$ yield.<smiles>COC(=O)[C@H](N)Cc1ccc(OC)c(B(O)O)c1</smiles>

Scheme 3. (a) EDCI, HOBt, DIPEA, DCM, RT, 2h, 85\%.

Compound 8 was coupled with compound 9 using 1-ethyl-3-(3-dimethylaminopropyl) carbodiimide hydrochloride and $N$-hydroxy benzotriazol in presence of diisopropyl ethyl amine in dichloromethane at room temperature to afford dipeptide $\mathbf{1 0}$ in $85 \%$ yield.
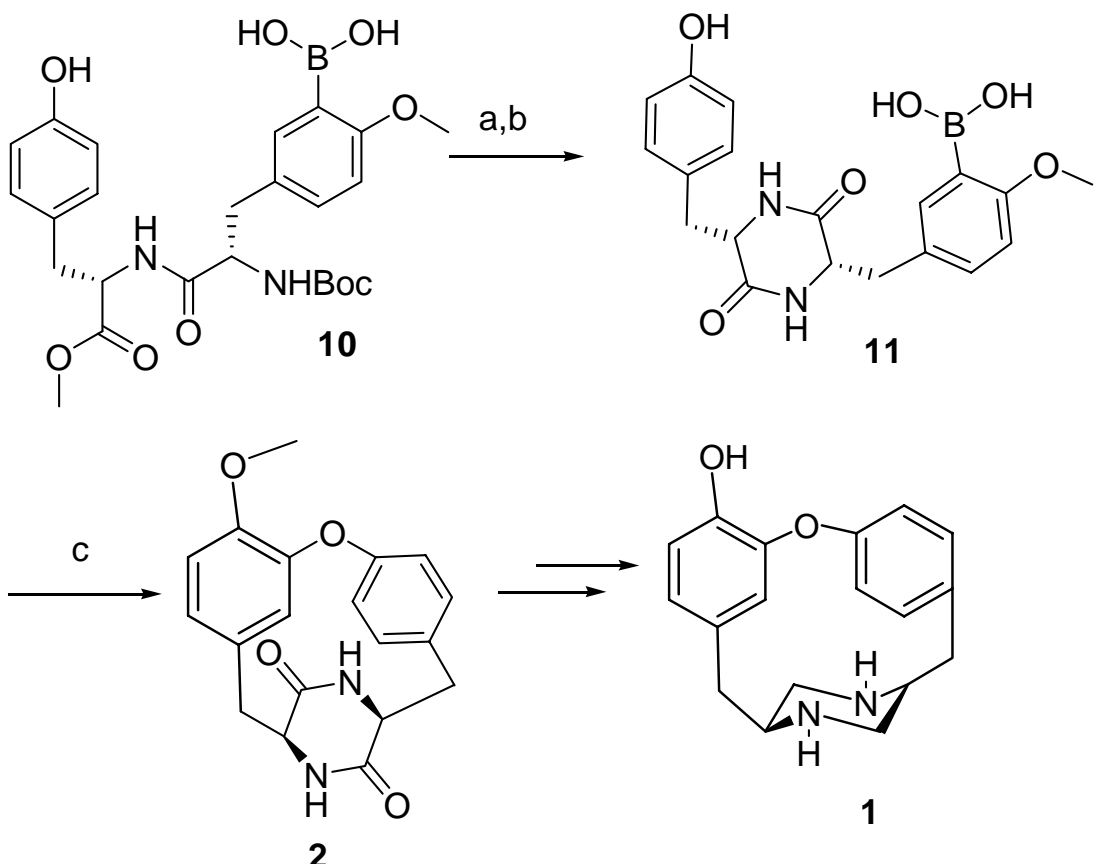

Scheme-4. (a) 2N HCl, EtOAc, RT, 30 min, $96 \%$, (b) 0.1M AcOH-iPrOH, NMM, 4h, reflux, 90 $\%$. (c) $\mathrm{Cu}(\mathrm{OAc})_{2}, \mathrm{Et}_{3} \mathrm{~N}, \mathrm{DMF}$, powdered $4 \mathrm{~A}^{0}$ molecular sieves, $72 \mathrm{~h}, 33 \%$. 
Compound 10 was $\mathrm{N}$-deprotected using $2 \mathrm{~N} \mathrm{HCl}$ in ethyl acetate to yield the corresponding hydrochloride salt which was then treated with $0.1 \mathrm{M}$ acetic acid in isopropyl alcohol in presence of $N$-methylmorpholine to afford compound $\mathbf{1 1}$ in $90 \%$ yield. Key ring closure of $\mathbf{1 1}$ to $2^{11}$ via the intramolecular $O$-arylation of phenol with arylboronic acid $\mathbf{1 1}$ smoothly proceeded using cupric acetate (1.3 eq.) and triethyl amine (5 eq.) in $N, N$-dimethyl formamide in presence of powered $4 \mathrm{~A}^{0}$ molecular sieves for $72 \mathrm{~h}$ at room temperature in $33 \%$ yield. The conversion of compound $\mathbf{2}$ to compound $\mathbf{1}$ has already been reported in the literature. ${ }^{12}$

\section{Conclusions}

Our methods described above provide new and shorter routes for the synthesis of piperazinomycin from commercially available L-tyrosine.

\section{Experimental Section}

General Procedures. All solvents and reagents were purchased from the suppliers and used without further purification. All non-aqueous reactions were performed in dry glassware under dry nitrogen atmosphere. Organic solutions were concentrated under reduced pressure. Thin layer chromatography was performed on Merck precoated Silica-gel $60 \mathrm{~F}_{254}$ plates. ${ }^{1} \mathrm{H}$ and ${ }^{13} \mathrm{C}$ NMR spectra were recorded on a Varian Gemini 400 MHz FT NMR spectrometer using DMSO$\mathrm{d}_{6}$ and $\mathrm{CDCl}_{3}$ as a solvent. The chemical shifts were reported in $\delta \mathrm{ppm}$ relative to TMS. The mass spectra were recorded on Shimadzu LCMS-QP 800 LC-MS and AB-4000 Q-trap LCMS/MS. Melting points were obtained by using the open capillary method and are uncorrected.

(S)-Methyl 3-(3-bromo-4-methoxyphenyl)-2-(tert-butoxycarbonyl) propanoate (5). To a solution of compound $4(5 \mathrm{~g}, 14.66 \mathrm{mmol})$ in $\mathrm{MeOH}(30 \mathrm{~mL})$ and EtOAc $(30 \mathrm{~mL})$ at room temperature was added $\mathrm{NaHCO}_{3}(2.45 \mathrm{~g}, 29.32 \mathrm{mmol})$ and $\mathrm{H}_{2} \mathrm{O}(20 \mathrm{~mL})$ followed by $(\mathrm{Boc})_{2} \mathrm{O}$ $(4.8 \mathrm{~g}, 22 \mathrm{mmol})$. The reaction mixture was stirred at same temperature for $4 \mathrm{~h}$. To the reaction mixture heptane $(50 \mathrm{~mL})$ was added and diluted with water $(20 \mathrm{~mL})$. The organic layer was separated and aqueous layer was washed with heptane $(40 \mathrm{~mL})$ one more time. Then the aqueous layer was acidified with $10 \%$ aqueous citric acid solution $(20 \mathrm{~mL})$ to make the $\mathrm{PH} \sim 4-5$. The aqueous layer was extracted with EtOAc $(2 X 50 \mathrm{~mL})$ and concentrated under vacuum to afford corresponding Boc-protected compound (5.2 g). To a solution of Boc-protected compound (5.2 $\mathrm{g}, 14.44 \mathrm{mmol})$ in acetone $(50 \mathrm{~mL})$ was added $\mathrm{K}_{2} \mathrm{CO}_{3}(6 \mathrm{~g}, 43.33 \mathrm{mmol})$ and $\mathrm{Me}_{2} \mathrm{SO}_{4}(4.5 \mathrm{~g}$, $36.11 \mathrm{mmol}$ ) at room temperature. The reaction mixture was stirred at same temperature for $12 \mathrm{~h}$. The reaction mixture was filtered through pad of Celite and washed with acetone $(50 \mathrm{~mL})$. The acetone layer was concentrated under vacuum. Purification by column chromatography (silica, 7:3 hexane/EtOAc) provided arylbromide 5 as an off white solid (on storing at rt slowly became 
solid) (5.5 g, 96\% yield), $\mathrm{R}_{\mathrm{f}}=0.6$ ( 7:3 ; heptanes/EtOAc), mp 66-70 ${ }^{\circ} \mathrm{C},{ }^{1} \mathrm{H}$ NMR (400 MHz, DMSO- $_{6}$ ): $\delta 7.40(\mathrm{~s}, 1 \mathrm{H}), 7.25(\mathrm{~d}, \mathrm{~J}=8.4 \mathrm{~Hz}, 1 \mathrm{H}), 7.18(\mathrm{dd}, \mathrm{J}=8.4 \mathrm{~Hz}, 1 \mathrm{H}), 6.99$ (d, J = 8.4 Hz, $1 \mathrm{H}), 4.08(\mathrm{~m}, 1 \mathrm{H}), 3.76(\mathrm{~s}, 3 \mathrm{H}), 3.57(\mathrm{~s}, 3 \mathrm{H}), 2.89(\mathrm{~m}, \mathrm{~J}=5.2 \mathrm{~Hz}, 1 \mathrm{H}), 2.75(\mathrm{~m}, \mathrm{~J}=10 \mathrm{~Hz}, 1 \mathrm{H})$, $1.28(\mathrm{~s}, 9 \mathrm{H})) ;{ }^{13} \mathrm{C}$ NMR (400 MHz, DMSO-d 6 ): $\delta$ 170.9, 158.9, 155.1, 134.2, 131.0, 130.6, $114.5,113.1,79.9,56.7,53.5,53.3,35.4,34.8 ;$ ESIMS: $\mathrm{m} / \mathrm{z}$ calcd $[\mathrm{M}+]$ : 388; found: 287.94 $[\mathrm{M}+]$.

(S)-Methyl 2-(tert-butoxycarbonyl)-3-(4-methoxy-3-(4， 4，5，5-tetramethyl-1， 3， 2dioxaborolan-2-yl) phenyl) propanoate (6). Aryl bromide 5 (5 g, $12.88 \mathrm{mmol}$ ), bis (pinacolato) diboron (4.23 g, $16.75 \mathrm{mmol})$, KOAc (3.78 g, $38.65 \mathrm{mmol})$ and $\mathrm{PdCl}_{2}$ (dppf) (0.525 g, 0.644 mmol) were suspended in dry 1, 2-dimethoxyethane $\left(50 \mathrm{~mL}\right.$, degassed by sparging with $\left.\mathrm{N}_{2}\right)$, and heated to $110{ }^{\circ} \mathrm{C}$ for $18 \mathrm{~h}$. Water $(30 \mathrm{~mL})$ and EtOAc $(30 \mathrm{~mL})$ were added. The layers were separated, and the aqueous layer was extracted with EtOAc $(2 \times 50 \mathrm{~mL})$. The combined organic layers were dried $\left(\mathrm{MgSO}_{4}\right)$, filtered, and concentrated in vacuum. Purification by column chromatography (silica, 7:3 hexane/EtOAc) provided aryl boronate $\mathbf{6}$ as an off white solid (on storing at rt slowly became solid) $(5 \mathrm{~g}, 90 \%$ yield $), \mathrm{R}_{\mathrm{f}}=0.5$ ( 7:3; heptanes/EtOAc), mp 92-95 ${ }^{\circ} \mathrm{C},{ }^{1} \mathrm{H}$ NMR (400 MHz, DMSO-d $\left.)_{6}\right): \delta 7.21-7.33(\mathrm{~m}, 3 \mathrm{H}), 6.82(\mathrm{~d}, \mathrm{~J}=8.8 \mathrm{~Hz}, 1 \mathrm{H}), 4.00(\mathrm{~m}, \mathrm{~J}=$ $8.8 \mathrm{~Hz}, 1 \mathrm{H}), 3.66(\mathrm{~s}, 3 \mathrm{H}), 3.56(\mathrm{~s}, 3 \mathrm{H}), 2.77-2.88(\mathrm{~m}, 2 \mathrm{H}), 1.29(\mathrm{~s}, 6 \mathrm{H}), 1.19(\mathrm{~s}, 6 \mathrm{H}), 1.03(\mathrm{~s}$, $9 \mathrm{H}) ;{ }^{13} \mathrm{C}$ NMR (400 MHz, DMSO-d 6 ): $\delta 172.9,160.0,155.1,133.3,131.9,131.3,123.3,111.2$, 85.3, 80.8, 56.9, 55.3, 51.3, 36.8, 29.3, 24.8, 23.9; ESIMS: m/z calcd [ $\mathrm{M}+]$ : 435; found: 436 [M+H+]; HRMS (ESI): m/z calcd [M+]: 435.3188; found: $435.3165[\mathrm{M}+]$.

(S)-5-(2-(tert-butoxycarbonyl)-3-methoxy-3-oxopropyl)-2-methoxyphenylboronic acid (7). To a stirred solution of the arylboronate $6(5 \mathrm{~g}, 11.5 \mathrm{mmol})$ in acetone $(100 \mathrm{~mL})$ was added $\mathrm{NH}_{4} \mathrm{OAc}$ (aq.) $(125 \mathrm{~mL}, 0.1 \mathrm{~N})$ and $\mathrm{NaIO}_{4}(7.34 \mathrm{~g}, 34.48 \mathrm{mmol})$ at room temperature. The mixture was stirred at room temperature for $24 \mathrm{~h}$. To the reaction mixture ethyl acetate $(50 \mathrm{ml})$ was added and organic layer was separated and concentrated. Purification by column chromatography (silica, 5:5 hexane/EtOAc) provided arylboronic acid 7 as an off white solid (on storing at rt slowly became solid) (3.6 g, 90\% yield ), $\mathrm{R}_{\mathrm{f}}=0.3$ ( 5:5; heptanes/EtOAc), mp 95$100{ }^{\circ} \mathrm{C},{ }^{1} \mathrm{H}$ NMR $\left(400 \mathrm{MHz}, \mathrm{CDCl}_{3}\right): \delta 7.53(\mathrm{~s}, 1 \mathrm{H}), 7.16(\mathrm{~d}, \mathrm{~J}=8.4 \mathrm{~Hz}, 1 \mathrm{H}), 6.79(\mathrm{~d}, \mathrm{~J}=8.8 \mathrm{~Hz}$, $1 \mathrm{H}), 6.28(\mathrm{~s}, 2 \mathrm{H}), 4.50(\mathrm{~m}, 1 \mathrm{H}), 3.82(\mathrm{~s}, 3 \mathrm{H}), 3.67(\mathrm{~s}, 3 \mathrm{H}), 3.02(\mathrm{~m}, \mathrm{~J}=5.6 \mathrm{~Hz}, 2 \mathrm{H}), 1.36(\mathrm{~s}$, $9 \mathrm{H})$ ); ${ }^{13} \mathrm{C} \mathrm{NMR}\left(400 \mathrm{MHz}, \mathrm{CDCl}_{3}\right): \delta 172.4,163.6,155.0,137.7,133.4,133.3,128.4,110.0$, 79.8, 55.5, 54.5, 52.2, 37.4, 28.9; ESIMS: $\mathrm{m} / \mathrm{z}$ calcd [ $\mathrm{M}+]: 353$; found: 354 [M+1].

(S)-3-(3-borono-4-methoxyphenyl)-2-(tert-butoxycarbonyl)propanoic acid (8). To a stirred solution of the arylboronic acid $7(3.6 \mathrm{~g}, 10.2 \mathrm{mmol})$ in $\mathrm{MeOH}(20 \mathrm{~mL})$ and $\mathrm{H}_{2} \mathrm{O}(20 \mathrm{~mL})$ was added $\mathrm{LiOH}(0.73 \mathrm{~g}, 30.6 \mathrm{mmol})$ at room temperature. The reaction mixture was stirred at same temperature for $30 \mathrm{~min}$. To the reaction mixture heptane $(30 \mathrm{~mL})$ was added and stirred for another $15 \mathrm{~min}$. The aqueous layer was separated and acidified with $10 \%$ aq. citric acid solution to make $\mathrm{PH} \sim 4$. The aqueous layer was extracted with EtOAc ( 3 X30 mL). The combined organic layers were dried $\left(\mathrm{MgSO}_{4}\right)$, filtered, and concentrated in vacuum to afford compound 8 as an off white solid (on storing at rt slowly became solid) $\left(3.3 \mathrm{~g}, 96 \%\right.$ yield), mp $98-102{ }^{\circ} \mathrm{C},{ }^{1} \mathrm{H}$ NMR (400 MHz, DMSO-d $\left.{ }_{6}\right): \delta 8.22(\mathrm{~s}, 2 \mathrm{H}), 7.37$ (s, 1H), $7.19(\mathrm{~m}, \mathrm{~J}=8 \mathrm{~Hz}, 1 \mathrm{H}), 6.98(\mathrm{~d}, \mathrm{~J}=8.4$ 
$\mathrm{Hz}, 1 \mathrm{H}), 6.82(\mathrm{~d}, \mathrm{~J}=8 \mathrm{~Hz}, 1 \mathrm{H}), 3.95(\mathrm{~m}, 1 \mathrm{H}), 3.70(\mathrm{~s}, 3 \mathrm{H}), 2.86(\mathrm{~m}, 1 \mathrm{H}), 2.70(\mathrm{~m}, 1 \mathrm{H}), 1.24(\mathrm{~s}$, $9 \mathrm{H})$ ); ${ }^{13} \mathrm{C}$ NMR (400 MHz, DMSO-d 6 ): $\delta$ 174.0, 162.7, 155.8, 136.6, 132.6, 131.5, 129.9, 110.5, 79.5, 56.4, 55.7, 36.0, 28.5; ESIMS: $\mathrm{m} / \mathrm{z}$ calcd [ $\mathrm{M}+]$ : 339; found: 340 [M+1].

\section{5-((S)-2-(tert-butoxycarbonyl)-3-((S)-3-(4-hydroxyphenyl)-1-methoxy-1-oxopropan-2-} ylamino)-3-oxopropyl)-2-methoxyphenylboronic acid (10). A suspension of 8 (3 g, 6.89 $\mathrm{mmol})$ in dry DCM $(50 \mathrm{~mL})$ was added compound 9 (1.35 g, $6.89 \mathrm{mmol})$, EDCI (1.97 g, 10.34 mmol), HOBt $(0.93 \mathrm{~g}, 6.89 \mathrm{mmol})$ and DIPEA (1.33 g $10.34 \mathrm{mmol})$ at room temperature. The resulting mixture was stirred at room temperature for $2 \mathrm{~h}$. The reaction mixture was quenched with water and neutralized with $10 \%$ aq. citric acid solution and dichloromethane layer was separated and concentrated to get a residue. The residue was purified by column chromatography (silica, 5:5; heptanes / EtOAc) to afford compound $\mathbf{1 0}$ as an off white solid (3.00 g, 85\% yield) as a white solid. $\mathrm{R}_{\mathrm{f}}=0.2$ ( 5:5; Heptane/EtOAc), mp 90-95 ${ }^{\circ} \mathrm{C},{ }^{1} \mathrm{H}$ NMR (400 MHz, DMSO-d 6 ): $\delta 9.21(\mathrm{~s}, 1 \mathrm{H}), 8.24(\mathrm{~d}, \mathrm{~J}=7.56 \mathrm{~Hz}, 1 \mathrm{H}), 7.65(\mathrm{~s}, 2 \mathrm{H}), 7.49(\mathrm{~s}, 1 \mathrm{H}), 6.64-7.23(\mathrm{~m}, 7 \mathrm{H}), 4.41$ (q, $1 \mathrm{H}), 4.09(\mathrm{q}, 1 \mathrm{H}), 3.77(\mathrm{~s}, 3 \mathrm{H}), 3.58(\mathrm{~s}, 3 \mathrm{H}), 2.79-2.88(\mathrm{~m}, 4 \mathrm{H}), 1.28(\mathrm{~s}, 9 \mathrm{H}) ;{ }^{13} \mathrm{C}$ NMR $(400$ MHz, DMSO-d $)_{6}: \delta 171.7,171.6,162.1,155.9,155.0,136.3,132.2,129.9,129.8,128.0,126.8$, 114.9, 109.9, 77.9, 56.2, 55.8, 55.2, 53.6, 36.5, 36.0, 28.0; ESIMS: m/z calcd [ M+]: 516; found: $517[\mathrm{M}+\mathrm{H}+]$; HRMS (ESI): $\mathrm{m} / \mathrm{z}$ calcd [M+]: 516.3485 ; found: $516.3435[\mathrm{M}+]$.

\section{5-(((2S,5S)-5-(4-hydroxybenzyl)-3,6-dioxopiperazin-2-yl)methyl)-2-methoxyphenylboronic}

acid (11). A solution of $10(2.5 \mathrm{~g}, 6.00 \mathrm{mmol})$ in $2 \mathrm{M} \mathrm{HCl-EtOAc}(50 \mathrm{~mL})$ was stirred at room temperature for $30 \mathrm{~min}$. The volatiles were removed in vacuum and the residue was dried thoroughly under vacuum to afford the corresponding amine hydrochloride salt as an off white solid. A suspension of the hydrochloride salt in $0.1 \mathrm{M} \mathrm{HOAc-iPrOH}(30 \mathrm{ml})$ was treated with $N$-methylmorpholine (NMM, $0.64 \mathrm{~g}, 6.34 \mathrm{mmol}, 1.3 \mathrm{equiv}$ ) at room temperature, and the resulting weakly acidic reaction mixture was heated at reflux for $4 \mathrm{~h}$. The diketopiperazine began to crystallize from the hot reaction solution. The mixture was cooled at $0^{0} \mathrm{C}(4 \mathrm{~h})$ and filtered, and the collected product was washed with $\mathrm{Et}_{2} \mathrm{O}(3 \mathrm{X} 30 \mathrm{~mL})$ to afford compound 11 (1.67 g, 90\% yield) as an off white solid. mp 222-225 ${ }^{\circ} \mathrm{C},{ }^{1} \mathrm{H}$ NMR (400 MHz, DMSO-d 6 ): $\delta$ $9.23(\mathrm{~s}, 1 \mathrm{H}), 7.80(\mathrm{~s}, 2 \mathrm{H}), 7.61(\mathrm{~s}, 2 \mathrm{H}), 7.35(\mathrm{~s}, 1 \mathrm{H}), 6.82-7.04(\mathrm{~m}, 4 \mathrm{H}), 6.64(\mathrm{~d}, \mathrm{~J}=8 \mathrm{~Hz}, 2 \mathrm{H})$, $3.86(\mathrm{~m}, 2 \mathrm{H}), 3.68(\mathrm{~s}, 3 \mathrm{H}), 3.02(\mathrm{~m}, 2 \mathrm{H}), 2.64(\mathrm{~m}, 2 \mathrm{H}) ;{ }^{13} \mathrm{C}$ NMR (400 MHz, DMSO-d 6 ): $\delta$ $166.2,166.1,162.5,156.0,136.9,133.0,130.7,128.1,126.4,114.9,113.6,110.1,55.7,55.3$, 52.7, 38.9, 38.5; ESIMS: m/z calcd [ $\mathrm{M}+]$ : 384; found: $385.11[\mathrm{M}+\mathrm{H}+], 406.92[\mathrm{M}++\mathrm{Na}]$; HRMS (ESI): $\mathrm{m} / \mathrm{z}$ calcd [M+]: 384.1908; found: 384.1935 [M+].

(3S, 6S)-11-Methoxy-5, 21-dioxo-13-oxa-4, 20 - diazatetracyclo- $\left[\begin{array}{llll}12.2 .2 .2^{3,} & { }^{6} 1^{8,} & 12\end{array}\right]$ heniecosa-8, 10, 12(19), 14, 16, 17-hexaen (2). To a stirred suspension of compound 11 ( $1 \mathrm{~g}$, $2.60 \mathrm{mmol})$, anhydrous $\mathrm{Cu}(\mathrm{OAc})_{2}(0.611 \mathrm{~g}, 3.38 \mathrm{mmol})$ and activated $4 \AA$ molecular sieves $(2 \mathrm{~g})$ in dry DMF $(50 \mathrm{~mL})$ was added triethyl amine $(1.8 \mathrm{ml}, 13.02 \mathrm{mmol})$ at room temperature. The mixture was stirred vigorously for $74 \mathrm{~h}$ at room temperature, then filtered through a pad of Celite, and washed with water $(15 \mathrm{~mL})$ and $10 \% \mathrm{MeOH}-\mathrm{CHCl}_{3}(20 \mathrm{ml})$. The organic layer was separated and dried over $\mathrm{MgSO}_{4}$ and was evaporated to dryness in vacuum. The residue was subjected to column chromatography (silica, 2-10\% $\left.\mathrm{CH}_{3} \mathrm{OH} / \mathrm{CHCl}_{3}\right)$ to afford compound $2(0.29$ 
g, 33\%) as a white solid: $\mathrm{R}_{\mathrm{f}}=0.3\left(9: 1 ; \mathrm{CHCl}_{3} / \mathrm{MeOH}\right) ; \mathrm{mp} 280-282^{\circ} \mathrm{C},[\alpha]^{25}{ }_{\mathrm{D}}+182$ (c 0.05 , $\mathrm{MeOH}){ }^{1} \mathrm{H}$ NMR (400 MHz, DMSO-d 6 ): $\delta 8.09$ (s, 1H), $7.89(\mathrm{~s}, 1 \mathrm{H}), 6.45-7.40(\mathrm{~m}, 6 \mathrm{H}), 4.27$ $(\mathrm{m}, 1 \mathrm{H}), 4.20(\mathrm{~m}, 1 \mathrm{H}), 4.14(\mathrm{~d}, 1 \mathrm{H}), 3.83(\mathrm{~s}, 3 \mathrm{H}), 3.05-3.48(\mathrm{~m}, 2 \mathrm{H}), 2.87-2.72(\mathrm{~m}, 2 \mathrm{H}) ;{ }^{13} \mathrm{C}$ NMR (400 MHz, DMSO-d $\left.{ }_{6}\right): \delta$ 166.9, 166.3, 157.8, 153.1, 145.8, 133.5, 133.0, 131.8, 128.9, 125.0, 114.9, 112.7, 56.0, 55.0, 51.7, 36.5, 31.4; ESIMS: m/z calcd [ $\mathrm{M}+]$ : 338; found: 339 [M+H+]; HRMS (ESI): m/z calcd [M+]: 338.3572; found: $338.3385[\mathrm{M}+]$.

\section{Acknowledgements}

We are grateful to Indian Association for Cultivation Sciences, Jadavpur for analytical support, Pfizer Ltd, and SVNIT, Surat, India.

\section{References}

1. Jolad, S. D.; Hoffmann, J. J.; Torrance, S. J.; Wiedhopf, R. M.; Cole, J. R.; Arora, S. K.; Bates, R. B.; Gargiulo, R. L.; Kriek, G. R. J. Am. Chem. Soc. 1977, 99, 8040.

2. Itokawa, H.; Morita, H.; Takeya, K.; Tomioka, N.; Itai, A. Chem. Lett. 1991, 2217.

3. Sano, S.; Ikai, K.; Katayama, K.; Takesato, K.; Nakamura,T.; Obayashi, A.; Ezure, Y.; Enomoto, H. J. Antibiot. 1986, 39, 1685.

4. Kase, H.; Kaneko, M.; Yamada, K. J. Antibiot. 1987, 40, 450.

5. Chan, D. M. T.; Monaco, K. L.; Wang, R.-P.; Winters, M.P. Tetrahedron Lett. 1998, 39, 2933.

6. Evans, D. A.; Katz, J. L.; West, T. R. Tetrahedron Lett. 1998, 39, 2937.

7. Lam, P. Y. S.; Clark, C. G.; Saubern, S.; Adams, J.; Winters, M. P.; Chan, D. M. T.; Combs, A. Tetrahedron Lett. 1998, 39, 2941.

8. Jung, M. E.; Lazarova, T. I. J. Org. Chem. 1999, 64, 2976.

9. Prieto, M.; Mayor, S.; Rodrı'guez, K.; Lloyd-Williams, P.; Ernest, G. J. Org. Chem. 2007, $72,1047$.

10. Coutts, S. J.; Adams, J.; Dale Krolikowski, D.; Snow, R. J. Tetrahedron Lett. 1994, 35, 5109.

11. Jacobsen, M. F.; Knudsen, M. M.; Gothelf, K. V. J. Org. Chem. 2006, 71, 9183.

12. Boger, D. L.; Zhou, J. J.Am. Chem. Soc. 1993, 115, 11426. 